\title{
Intrathecal administration of autologous mesenchymal stromal cells for spinal cord injury: Safety and efficacy of the 100/3 guideline
}

\author{
JESÚS VAQUERO ${ }^{1}$, MERCEDES ZURITA ${ }^{1}$, MIGUEL A. RICO $^{1}$, CONCEPCION AGUAYO $^{1}$, \\ CELIA BONILLA ${ }^{1}$, ESPERANZA MARIN ${ }^{2}$, NOEMI TAPIADOR $^{3}$, MARTA SEVILLA $^{3}$, \\ DAVID VAZQUEZ ${ }^{4}$, JOAQUIN CARBALLIDO ${ }^{4}$, CECILIA FERNANDEZ $^{1}$,

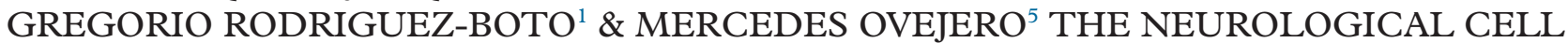 \\ THERAPY GROUP FROM PUERTA DE HIERRO-MAJADAHONDA HOSPITAL *
}

\author{
${ }^{1}$ Neurosurgery Service, University Hospital Puerta de Hierro-Majadahonda, Autonomous University, Madrid, Spain, \\ ${ }^{2}$ Clinical Neurophysiology Service, University Hospital Puerta de Hierro-Majadahonda, Autonomous University, \\ Madrid, Spain, ${ }^{3}$ Rehabilitation Service, University Hospital Puerta de Hierro-Majadahonda, Autonomous University, \\ Madrid, Spain, ${ }^{4}$ Urology Service, University Hospital Puerta de Hierro-Majadahonda, Autonomous University, \\ Madrid, Spain, and ${ }^{5}$ Statistic Unit, Sermes Contract Research Organization (CRO), Madrid, Spain
}

\begin{abstract}
Background aims. Cell therapy with autologous mesenchymal stromal cells (MSCs) in patients with spinal cord injury (SCI) is beginning, and the search for its better clinical application is an urgent need. Methods. We present a phase 2 clinical trial in patients with chronic SCI who received three intrathecal administrations of $100 \times 10^{6}$ MSCs and were followed for 10 months from the first administration. Efficacy analysis was performed on nine patients, and safety analysis was performed on 11 patients. Clinical scales, urodynamic, neurophysiological and neuroimaging studies were performed previous to treatment and at the end of the follow-up. Results. The treatment was well-tolerated, without any adverse event related to MSC administration. Patients showed variable clinical improvement in sensitivity, motor power, spasms, spasticity, neuropathic pain, sexual function or sphincter dysfunction, regardless of the level or degree of injury, age or time elapsed from the SCI. In the course of follow-up three patients, initially classified as ASIA A, B and C, changed to ASIA B, C and D, respectively. In urodynamic studies, at the end of follow-up, $66.6 \%$ of the patients showed decrease in postmicturition residue and improvement in bladder compliance. At this time, neurophysiological studies showed that $55.5 \%$ of patients improved in somatosensory or motor-evoked potentials, and that $44.4 \%$ of patients improved in voluntary muscle contraction together with infralesional active muscle reinnervation. Conclusions. The present guideline for cell therapy is safe and shows efficacy in patients with SCI, mainly in recovery of sphincter dysfunction, neuropathic pain and sensitivity.
\end{abstract}

Key Words: cell therapy, mesenchymal stromal cells, neuropathic pain, sphincter dysfunction, spinal cord injury

\section{Introduction}

At present, cell therapy using autologous mesenchymal stromal cells (MSCs) is configured as a hope to improve the quality of life in patients with spinal cord injury (SCI) [1-12]. However, these new techniques are still in their infancy and it is necessary to know many details, such as the criteria to select the patients that can have better benefit, or to know dosage or administration guidelines. Based on previous experiences in animal models and in humans, intrathecal or intralesional administration of MSCs are a safe and useful strategy to achieve benefit in SCI, with the con- dition that the spinal cord is not anatomically sectioned $[11,12]$, which implies a careful study with magnetic resonance imaging (MRI) of each SCI [13]. In this same line of knowledge, our previous experience suggests that autologous MSCs are superior to the use of allogeneic MSCs, and that use of autologous plasma as a support for MSCs is better than saline [11]. On the other hand, previous studies in patients suggest that the benefit of this type of cell therapy is not related to the greater or lesser time of chronicity of the SCI. Furthermore, at least in certain efficacy parameters, there seems to exist a relationship between the benefit and the number of cells administered [11].

${ }^{\star}$ A complete list of the investigators (Neurological Cell Therapy Group from Puerta de Hierro-Majadahonda Hospital) is provided in the Supplementary Material.

Correspondence: Jesús Vaquero, MD, PhD, Neurosurgery Service, University Hospital Puerta de Hierro-Majadahonda, Autonomous University, Manuel de Falla, 1. 28222-Majadahonda, Madrid, Spain. E-mail: jvaqueroc@felefonica.net 
To obtain more experience in the possibilities of different guidelines of MSC administration in patients with SCI, here we present the results of a phase 2 clinical trial that analyzes the efficacy and safety of intrathecal administration of autologous MSCs with a guideline of three doses of 100 million MSCs, with an interval of 3 months between each administration (ClinicalTrials.gov NCT02570932, EudraCT: 2014-005613-24, Spanish Agency of Medicament and Health Products (AEMPS) number: 15-0253).

\section{Methods}

\section{Cell therapy medicament}

We used the NC1 medicament. It is a cell therapy medicament developed after pre-clinical studies by our group and currently approved as a medicament under clinical investigation (PEI number: 12-141) by the AEMPS. The medicament consists of autologous MSCs and autologous plasma as its excipient. Previous to $\mathrm{NC} 1$ preparation, a sample of peripheral blood was retrieved from each patient for genomic studies to rule out chromosomal abnormalities that could discourage cell expansion, and to obtain a genetic fingerprint (KaryoNIM Stem Cells and KaryoNIM STR test, respectively; NIMGenetics).

The genetic studies included the following: (1) analysis of the cellular genome by techniques Array comparative genomic hybridization (CGH) to ensure that the starting cells are genetically stable and the expansion process produces no genetic modification, and (2) analysis of genetic fingerprinting (Short Tandem Repeat [STR]) with the aim of having no crosscontamination with other cells in the manufacturing process. The genetic fingerprint analysis was done by the external laboratory NIMGenetics, with a panel of 20 markers + amelogenin. The panel was amplified by multiplex polymerase chain reaction (PCR) using the PCR kits PlusTM AmpFlSTR Identifiler Amplification Kit and/or PCR NGM AmpFISTR Amplification Kit (NIMGenetics). The analysis by capillary electrophoresis of the amplified products was performed on an ABI automated sequencer 3100 - Avant.

For the analysis of the profiles obtained, Gene Mapper software v 3.2.1 was used. The analysis of possible genetic abnormality was performed using a platform CGH Array (KaryoNIM STEM, NIMGenetics) optimized for use in cell therapy projects. This platform consists of 60000 probes distributed throughout the genome with a probe designed for each $60 \mathrm{~kb}$ and enables efficient detection of amplifications and deletions $>200 \mathrm{~Kb}$ and analysis in detail of 407 genes related to genomic instability and abnormal proliferation in accord with the Cancer Gene Census list. It has an enrichment probe designed specifically for the detection of 395 cancer-related genes, included in the Cancer Gene Census list (genes for which have been described causal mutations involved in cancer) with an average of five probes per gene. The platform includes 15 specific probes for detecting each of the 23 oncogenes commonly used in clinical diagnosis. In addition, there are probes designed specifically for the detection of six genes associated with stem cells. The minimum degree of mosaicism detected by array CGH is $20 \%-30 \%$. The scanning process allows the use of arrays $8 \times 60 \mathrm{k}$ with a resolution of $2 \mu \mathrm{m}$.

For obtaining the excipient, as a first step in the preparation of the NC1, we start with the removal of $500 \mathrm{~mL}$ of peripheral blood from each patient. In our cleanroom, blood was centrifuged at $900 \mathrm{~g}$ for $8 \mathrm{~min}$ to obtain the plasma fraction, which is aliquoted in $15-\mathrm{mL}$ tubes and stored at $-80^{\circ} \mathrm{C}$ until the medicament formulation.

Approximately 2 weeks later, $50 \mathrm{~mL}$ of bone marrow was aspirated under aseptic conditions from the iliac bones of each patient, immediately anticoagulated by a $5 \mathrm{~mL}$ solution composed of $100 \mathrm{IU} /$ $\mathrm{mL}$ sodium heparin Chiesi (ChiesiEspaña, L'Hospitalet de Llobregat, Spain) and $10^{4} \mathrm{IU} / 10^{4} \mu \mathrm{g}$ penicillinstreptomycin (BioWhittaker-Lonza) and sent to our cleanroom for culture and expansion under good manufacturing practice (GMP).

Mononuclear cells (MNCs) were separated by density gradient, using an automated cellprocessing system (SEPAX; BioSafe). Then, they were plated at a density of $16 \times 10^{4}$ to $20 \times 10^{4}$ cells $/ \mathrm{cm}^{2}$, in $175-$ $\mathrm{cm}^{2}$ flasks on Alpha-Minimum Essential Medium (MEM) with Earle's Balanced Salt Solution (BSS), and supplemented with $20 \%$ prion-free LGC standard serum (SLU ATCC.SCRR-3020, lot 63753841; Salvador Spriu) and $10^{4} \mathrm{IU} / 10^{4} \mu \mathrm{g}$ penicillinstreptomycin (BioWhittaker-Lonza). The cultures were maintained at $37^{\circ} \mathrm{C}$ in a humidified $5 \% \mathrm{CO}_{2}$ atmosphere for 3 days, after which nonadherent cells were removed by replacing the medium. When the cultures approached confluence $(90 \%-100 \%)$, adherent cells were detached by treatment with trypsin/ ethylenediaminetetraacetic acid (EDTA) solution (BioWhittaker-Lonza). Neutralization of trypsin and subsequent washing were performed with AlphaMEM medium supplemented with $10 \%$ fetal bovine serum (FBS) and $2 \mathrm{mmol} / \mathrm{L} \mathrm{L}$-glutamine, centrifuging at $1250 \mathrm{rpm}$ for $10 \mathrm{~min}$. After study of viability, cells were cultured to obtain the required number according to the plan previously made for each patient. Cells were replated at a density of 3000-5000 cells/ $\mathrm{cm}^{2}$ in factory farming of four floors with freeantibiotic Alpha-MEM medium supplemented with $10 \% \mathrm{FBS}$ and $2 \mathrm{mmol} / \mathrm{L} \mathrm{L}$-glutamine, and the culture was maintained renewing the medium every 3-4 days until there was a confluence of $90 \%-100 \%$. Once the 
culture reached confluency, it was prepared to obtain the bulk of MSCs. At this time, MSCs were detached with trypsin/EDTA and washed with Hank's BSS medium (BioWhittaker-Lonza) supplemented with $5 \%$ albumin (20\% albumin Grifols). After this, MSCs were resuspended with the previously obtained autologous plasma to remove traces of the washing medium. After cell counting, MSCs for the successive doses were separated and then cryopreserved, in $1.8 \mathrm{~mL}$ cryotubes at a concentration of $2.2 \times 10^{6}$ cells/ $\mathrm{mL}$, in a FBS solution with $10 \%$ dimethylsulphoxide (DMSO; Miltenyi Biotec). For it, we used the standard technique of cryopreservation in an isopropanol chamber. Finally, the MSCs for surgical administration were formulated, according to the number scheduled for each patient, after a new centrifugation at $1250 \mathrm{rpm}$ for $10 \mathrm{~min}$.

To prepare the successive doses, cryopreserved MSCs were thawed in a thermostatic bath at $37^{\circ} \mathrm{C}$, washed with antibiotic-free Alpha-MEM medium supplemented with $10 \% \mathrm{FBS}$ and $2 \mathrm{mmol} / \mathrm{L}$ L-glutamine and centrifuged at $1250 \mathrm{rpm}$ for $10 \mathrm{~min}$. After this, a cell count was performed and MSCs were plated at a concentration of 10 000-15 000 MSCs/ $\mathrm{cm}^{2}$ in $175-\mathrm{cm}^{2}$ culture flasks with antibiotic-free Alpha-MEM medium supplemented with 10\% FBS and $2 \mathrm{mmol} / \mathrm{L}$ L-glutamine, to reach a confluence of $90 \%-100 \%$ during a period of $4-5$ days, and then we proceeded in the same way as with the first MSC dose.

\section{Formulation and packaging}

After obtaining the MSCs for first or successive dose administration, they were resuspended in the autologous plasma at a cell concentration of $10^{4}$ cells $/ \mu \mathrm{L}$. After formulation, the cell therapy medicament was packaged in sterile and endotoxins-free 1-mL Hamilton microsyringes, with a 20-gauge needle. Subsequently the needle was removed and a sterile luer plug nut was placed on the end of each preloaded syringe. Microsyringes with the medicament were placed inside a sterile metal box, which was also double bagged before being transported for cell administration.

\section{Phenotypic characterization of MSCs}

For phenotypic characterization of MSCs, monoclonal antibodies conjugated with different fluorochromes (Fluorescein [FITC]/Phycoerythrin [PE]/Alexa-647 [AL-647]), which combine a number of both positive and negative MSC membrane markers, were used. Positive markers used were CD105 FITC (R\&D Systems); CD90 AL-647 (AbDSerotec,); HLA Class I FITC (Cytognos); CD73 PE (BD Bioscience) and CD166 PE (R\&D Systems). Negative markers used were CD34 PE (BD Bioscience); HLA class IIPE (Cytognos); CD80 AL-647 (AbDSerotec); CD45
FITC (Cytognos) and CD31 FITC (Cytognos). Furthermore, suitable isotopic controls for FITC, PE (Cytognos) and AL-647 (AbDSerotec) were used as controls for specificity of the monoclonal antibodies.

The labelled cells were acquired with a flow cytometer FC500 MPL Cytomics (Beckman Coulter) using the MXP software (Beckman Coulter). Nonviable cells were discarded using the labeling reagent LIVE\&DEAD (Invitrogen), and the collected data were analyzed with the CXP analysis software, version 2.1 (Beckman Coulter). Criteria for the administration of MSCs in our present clinical trial included a viability $>95 \%$, absence of microbial contamination (bacteria, fungus, virus or mycoplasma), expression of CD105, CD90, HLA I, CD73 and CD166 for $>90 \%$ of cells and absence of CD34, CD80, HLA II, CD45 and CD31 (expression of each $<5 \%$ ), as assessed using flow cytometry (Figure 1).

\section{Study design and treatment}

The clinical trial protocol was approved by the ethics committee of Puerta de HierroMajadahonda Hospital and by the AEMPS. It was conducted in accordance with the principles of the Declaration of Helsinki [14] and good clinical practice guidelines [15]. A flow chart of the patients can be seen in Figure 2. Adverse events (AEs) were collected throughout the follow-up and classified according to the Medical Dictionary for Regulatory Activities (MedDRA v. 18.1). This clinical trial studied 11 patients (male/female: 7/4). Age ranged between 28 and 62 years (mean \pm standard deviation [SD], $44.91 \pm 10.17$ years). The mean $\pm \mathrm{SD}$ of time elapsed from the time of SCI until the moment of initiating the cell therapy treatment was $13.65 \pm 14.79$ years. According the American Spinal Injury Association (ASIA) Impairment Scale (AIS) classification of SCI, three patients (27.27\%) were ASIA A, four patients $(36.36 \%)$ were ASIA B, three patients (27.27\%) were ASIA C and one patient $(9.09 \%)$ was ASIA D. With regard to the level of SCI, four patients $(36.36 \%)$ had the lesion at cervical level, four patients $(36.36 \%)$ at the dorsal level and three patients $(27.27 \%)$ at the dorsolumbar level.

Treatment consisted in the subarachnoid administration, by lumbar puncture, of $100 \times 10^{6}$ autologous MSCs obtained from bone marrow, expanded and supported in autologous plasma (month 1 of the study), which was repeated at months 4 and 7 , reaching a total administration of $300 \times 10^{6}$ MSCs for each patient. The patients were followed up until month 10 . Clinical scores were obtained from each patient by means of the following scales: the scale provided by ASIA [16], the SCI functional rating scale of the International Association of Neurorestoratology (IANR-SCIFRS scale) [17], the Visual Analog Scale (VAS) for the evaluation 


\section{PHENOTYPE OF MSC}
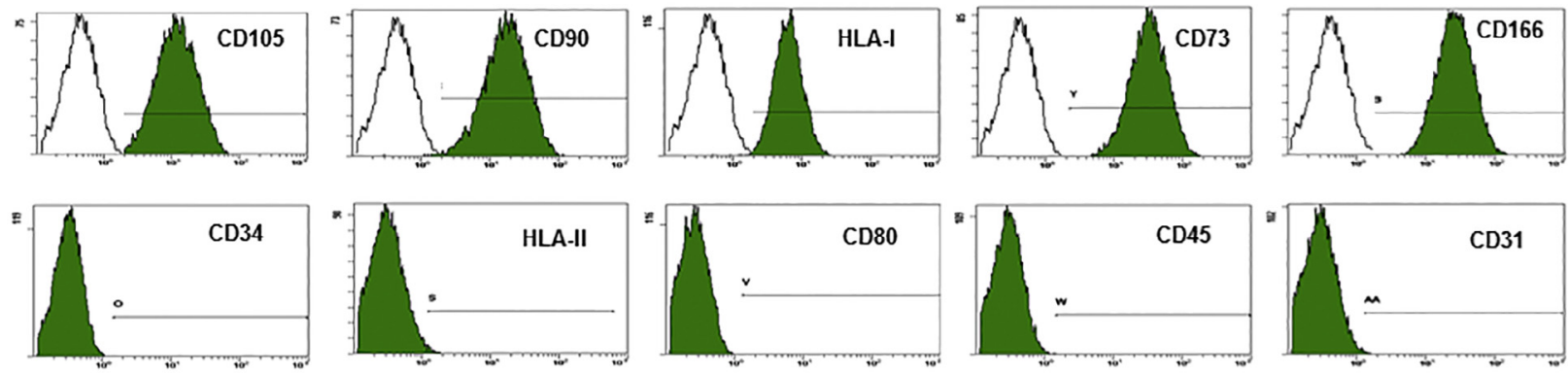

\begin{tabular}{|c|c|c|c|c|c|c|c|c|c|c|}
\hline MARKER & CD105 & CD90 & HLA-I & CD73 & CD166 & CD34 & CD80 & HLA-II & CD45 & CD31 \\
\hline $\begin{array}{c}\text { REFERENCE } \\
\text { PHENOTYPE }\end{array}$ & POSITIVE & POSITIVE & POSITIVE & POSITIVE & POSITIVE & NEGATIVE & NEGATIVE & NEGATIVE & NEGATIVE & NEGATIVE \\
\hline $\begin{array}{c}\text { PHENOTYPE } \\
\text { FOUND }\end{array}$ & POSITIVE & POSITIVE & POSITIVE & POSITIVE & POSITIVE & NEGATIVE & NEGATIVE & NEGATIVE & NEGATIVE & NEGATIVE \\
\hline CRITERION & $95 \% \pm 5 \%$ & $95 \% \pm 5 \%$ & $95 \% \pm 5 \%$ & $95 \% \pm 5 \%$ & $95 \% \pm 5 \%$ & $2.5 \% \pm 2.5 \%$ & $2.5 \% \pm 2.5 \%$ & $2.5 \% \pm 2.5 \%$ & $2.5 \% \pm 2.5 \%$ & $2.5 \% \pm 2.5 \%$ \\
\hline
\end{tabular}

Figure 1. Phenotypic characterization of the MSCs in our cell therapy medicament. MSCs showed expression of CD105, CD90, HLA I, CD73 and CD166 for $>90 \%$, and showed absence of CD34, CD80, HLA II, CD45 and CD31 expression ( $<5 \%$ for each).

of neuropathic pain [18], the Penn [19] and the modified Ashworth [20] scales for the evaluation of spasms and spasticity, respectively, the Geffner scale [21] for the study of bladder function and the Neurogenic Bowel Dysfunction (NBD) scale [22]. Neurophysiological, urodynamic and MRI studies were also performed before and after treatment (additional information is provided in the Supplementary Material).

\section{Statistical analysis}

To study the differences between the scores of the clinical scales, the nonparametric Wilcoxon rank test was used, comparing the result of each time period with results at baseline. In the results deemed statistically significant, the size of the effect was calculated using Cohen's d for paired samples, and the cut-offs proposed by Cohen [23] were used for the general interpretation of the cut-offs of this statistic. Descriptive analysis was performed for urodynamic and neurophysiological parameters. Safety analysis was analyzed by means of frequencies and percentages.

Statistical analysis was performed using $\mathrm{R}$ studio software (v 1.1.383) [24]. Per patient plots were made using ggplot2 package from $\mathrm{R}$ software [25]. The graphs were made with the GraphPad Prism program for Windows (v. 5.04; GraphPad Software). All inferential procedures used $\alpha=0.05$ as the level of risk.

Efficacy analyses were performed in the per protocol set. Following International Conference on Harmonisation (ICH) E9 guidelines [26] this set was defined as patients who complete the study follow- ing the protocol. Applying this definition, two patients were excluded from the statistical analysis. Patient 04 was excluded because this patient received his second dose out of window because the number of MSCs did not meet the minimum established by protocol. This patient received the second dose in month 8 (and by protocol this dose must be administered in month 4). This situation caused the patient to not be able to be compared with the other patients who did follow the protocol. Patient 05 was excluded because this patient left the study due to noncompliance with the protocol.

AEs were performed in the safety set that included all patients who received at least one dose of MSCs. Nevertheless, patient 04 was analyzed applying an $\mathrm{N}=1$ perspective and the results are presented as Supplementary Material. Therefore, efficacy analysis was performed on nine patients, and safety analysis was performed on 11 patients. Figure 2 shows the flow chart of the patients.

\section{Results}

In our present clinical trial, the cell expansion process did not involve any alteration to the genome of the cells in any of the cases, according to the results obtained after analysis using the Array-CGH platform.

$A E s$

AEs were grouped according to MedDRA terminology. During the study, of the 11 patients who were part of the Safety Population (PS), four of them had 


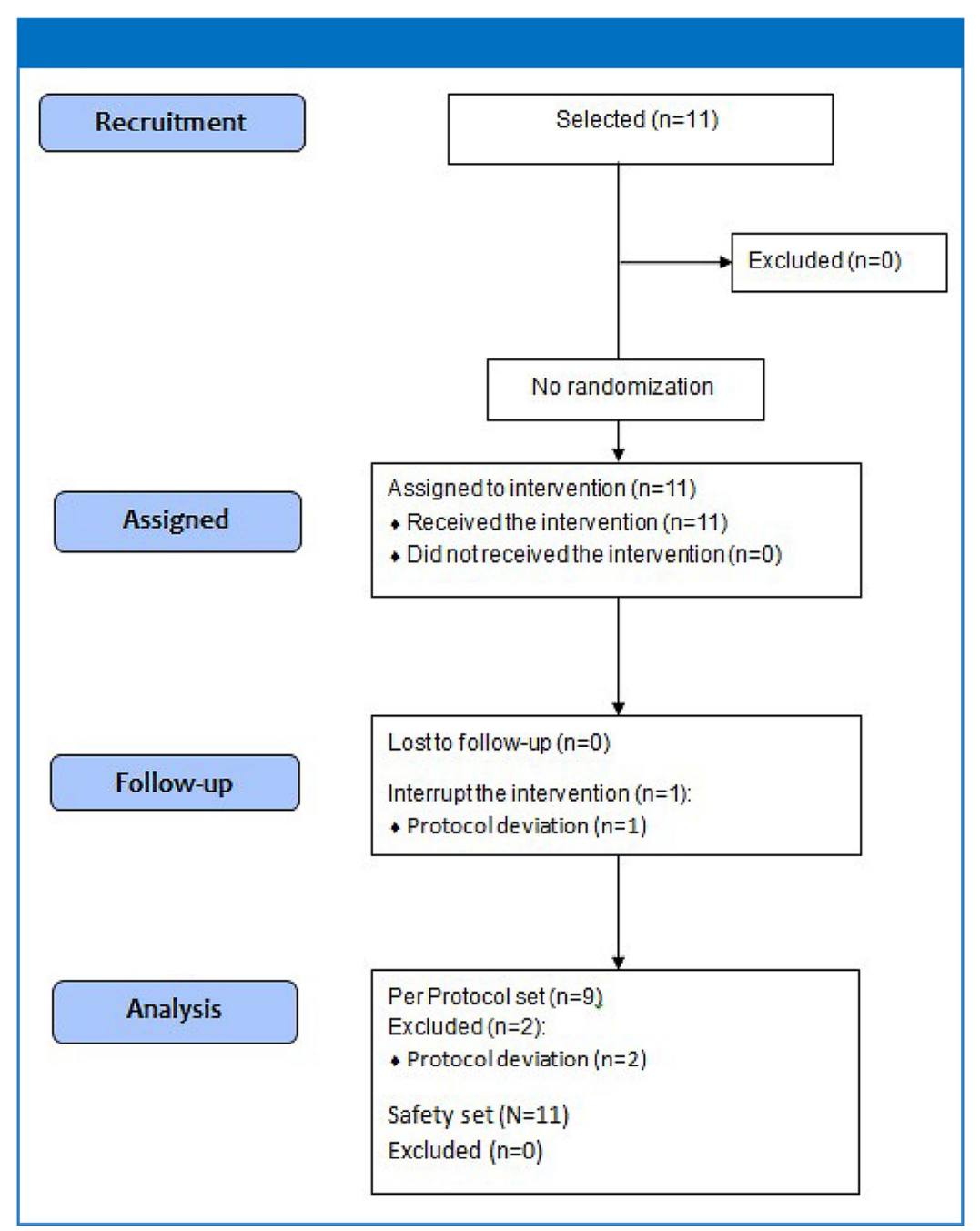

Figure 2. Flow chart of the study.

some type of AE, generally of mild intensity and collected as transitory sciatic pain $(37.5 \%)$, headaches and pain in the area of lumbar puncture. There was a severe $\mathrm{AE}$, unrelated to the treatment, that conditioned the withdrawal of the patient from the study.

\section{Sensitivity and motor improvement}

Statistically significant improvement in sensitivity according to the ASIA scale was already found in the first assessment performed after the first administration of cells, at month 4 of the study $(P=0.012$, effect size $(E S)=0.419$ for Pin Prick sensitivity, and $P=0.018, \mathrm{ES}=0.395$ for Light Touch sensitivity). The improvement was maintained during the study, with a $P$ value of $0.018(\mathrm{ES}=0.396)$ and 0.011 $(E S=0.426)$, respectively, at the end of the followup (Table I).

Motor score (MS) improvements were also observed during the study, with statistical significance from the fourth month $(P=0.048, \mathrm{ES}=0.330)$.
When improvements in MS of the four patients with SCI at cervical level were considered, we observed improvement in motor power of the upper extremities in three of them, and the fourth patient (patient 08), with a SCI at C7 level, did not show any modification. However, in the three patients showing MS improvement in upper extremities, more than two points of improvement for each muscle function examined was never observed (see Supplementary Material).

Furthermore, in the ASIA assessment performed in the fourth month of the study, one tetraplegic patient initially classified as ASIA A changed to ASIA B (patient 10), and in the ASIA assessment performed at the seventh month of the study, one patient initially classified as ASIA B changed to ASIA C (patient 06), and one patient initially classified as ASIA C (patient 07) changed to ASIA D (patient 07). Individual improvements in the ASIA assessment of the patients are provided in the Supplementary Material. 
Table I. Scores in ASIA scale.

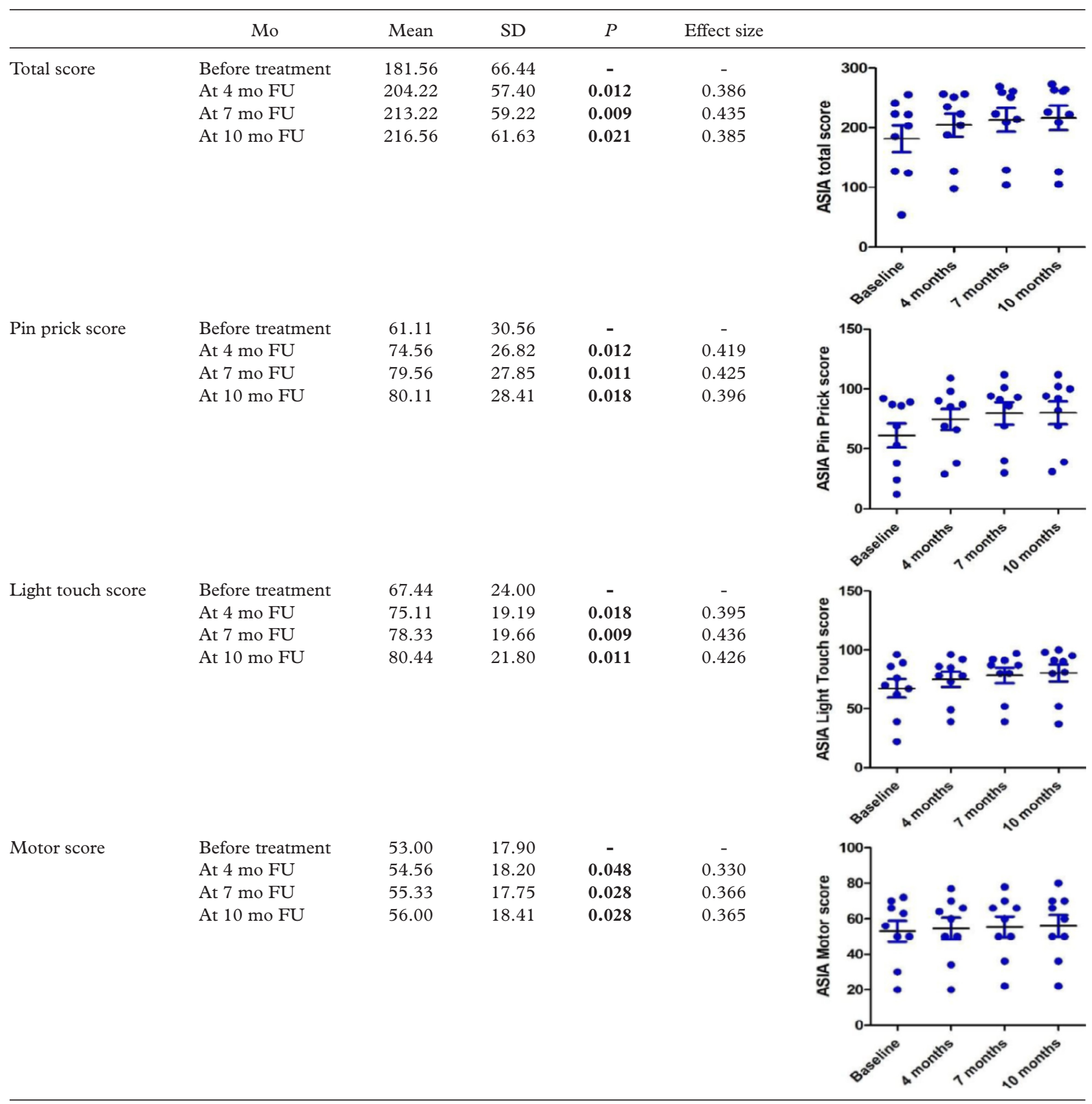

Significant $P$ values are highlighted in bold. Wilcoxon test was applied comparing each month with baseline. FU, follow-up.

However, in the ASIA assessment, the greater or lesser degree of improvements, both in motor power or in sensitivity, did not correlate with age, level, degree or chronicity of SCI.

\section{Spinal cord function assessed using the IANR-SCIFRS scale}

The IANR-SCIFRS scale evaluates the global spinal cord function through nine sections, with a final section that only applies to men and assesses sexual func- tion. When statistical study was performed, the mean score in global IANR-SCIFRS before treatment was 27.36 points (SD, 9.38 points) and, at end of the study, it was 36.20 points (SD, 5.71 points), showing a clear and statistically significant improvement $(P=0.009$, ES = 0.436; Table II).

The assessment of sexual function failed to obtain significant improvement throughout the study (only seven male patients were studied) but a tendency to improvement was observed. Nevertheless, a clear and significant improvement was observed in the 
Table II. Scores in IANR-SCIFRS scale.

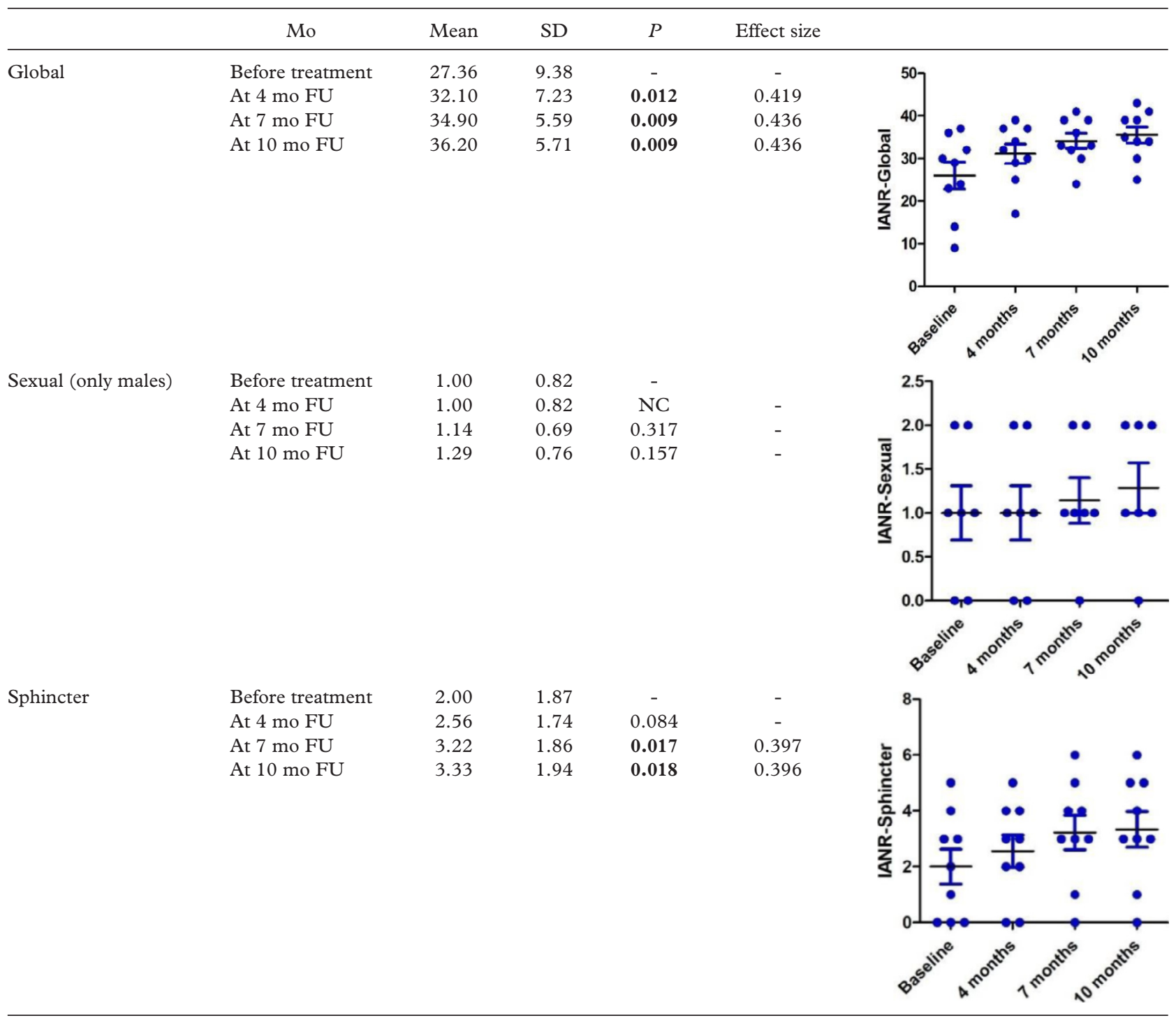

In IANR-Sexual $\mathrm{N}=7$. Significant $P$ values are highlighted in bold. Wilcoxon test was applied comparing each month with baseline. NC, non-computable.

assessment of sphincter function from the seventh month (with $P=0.018$ and $\mathrm{ES}=0.396$ at the end of the follow-up).

According to the analysis using the global IANRSCIFRS scale, before treatment, two patients of the series showed a "slight handicap", five patients showed a "medium handicap" and two patients showed a "severe handicap", whereas at the end of the followup, seven patients showed a "slight handicap" and the two remaining patients showed a "medium handicap" (Figure 3).

\section{Spasticity and spasms}

Prior to cell therapy treatment, six patients showed variable degree of spasticity and/or spasms, measured using the Ashworth and Penn scales. Both manifestations improved during the treatment, but at the end of the follow-up, no differences with statistical significance were found (Tables III and IV).

\section{Neuropathic pain}

Prior to cell therapy, neuropathic pain, as measured using the VAS scale, was present in eight patients. In all of them, it decreased or disappeared completely during the time of follow-up $(P=0.012, \mathrm{ES}=0.419)$, except in one patient in whom neuropathic pain was not modified. Table $\mathrm{V}$ shows the statistical analysis and evolution in the scores of neuropathic pain measured using the VAS scale. 


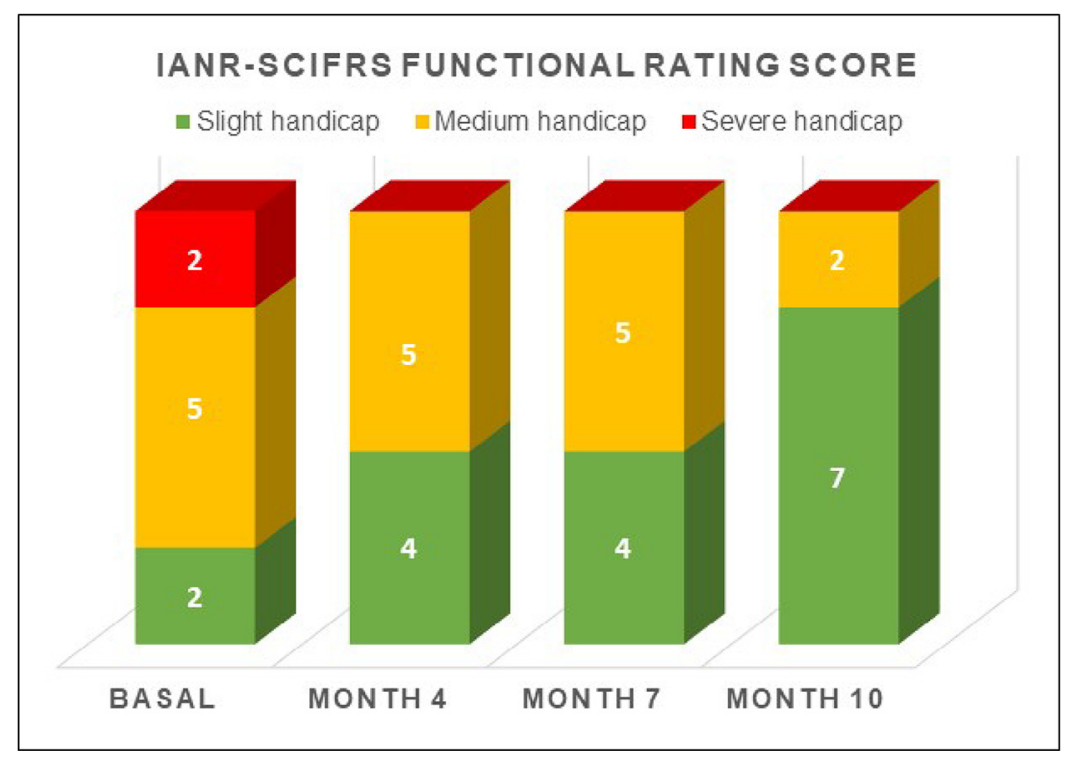

Figure 3. Evolution of the functional rating score of the patients, according to the IANR-SCIFRS scale. In this scale, a global score that ranged between 34 and 47 represents a slight handicap, between 17 and 33 represents a medium handicap and between 0 and 16 , a severe handicap. Previous to cell therapy two patients had slight handicap, five patients had medium handicap and two patients had severe handicap. At the end of the study, seven patients had slight handicap and two patients had medium handicap $(P=0.009, \mathrm{ES}=0.436)$.

\section{Effect of cell therapy on neurogenic bladder and bowel dysfunction}

We analyzed the possible improvement of bladder and bowel dysfunction using the scales of Geffner and NBD. In the Geffner scale, which measures bladder function, significant improvement was observed in our patients (with $P=0.028$ and $\mathrm{ES}=0.367$ from the seventh month of the follow-up; Table VI).

In the NBD scale, which values neurogenic bowel dysfunction, a clear improvement was observed from the first administration of MSCs (with $P=0.012$ and $\mathrm{ES}=0.419$ at the end of the follow-up; Table VII and Figure 4).

\section{Summary of clinical improvements}

Figure 5 summarizes the improvements obtained in the patients of the study. Patients 04 and 05 were not included in the Per Protocol set.

\section{Urodynamic studies}

According to the urodynamic studies, all the patients showed improvement at the end of the study in comparison with the studies carried out before the administration of cell therapy. Table VIII shows the improvements obtained for each patient in urodynamic parameters. The possibility of voluntary

Table III. Scores in Ashworth scale.

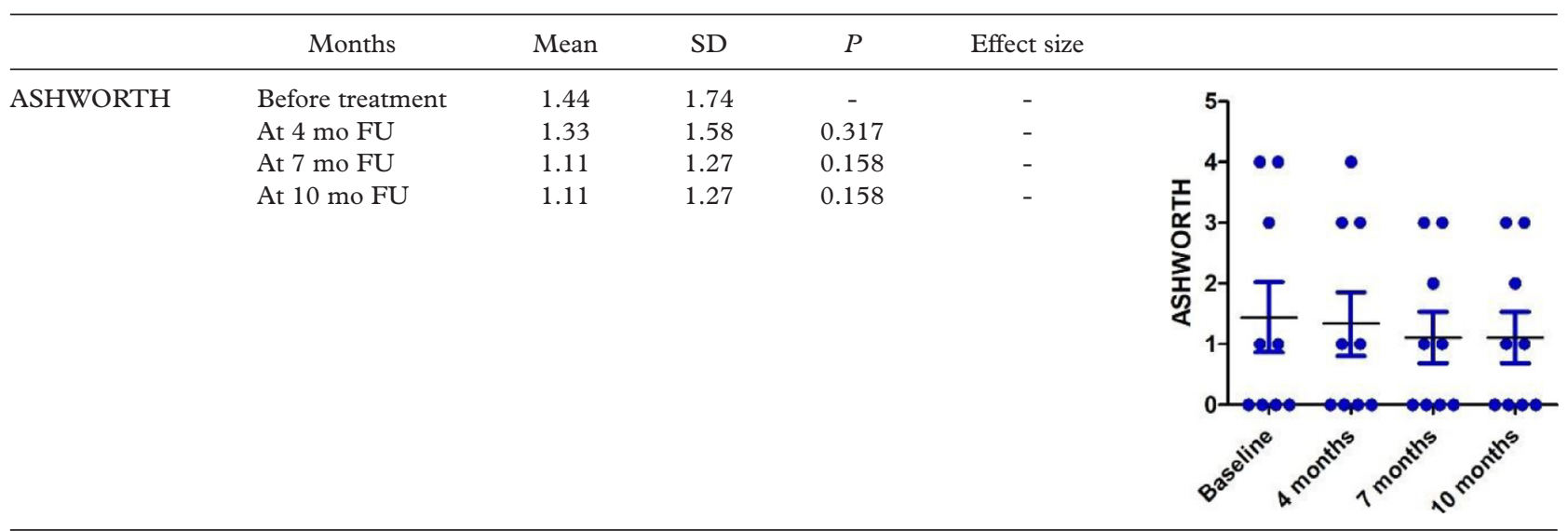

Wilcoxon test was applied comparing each month with baseline. Tendency to improvement was observed but without statistical significance. 
Table IV. Scores in Penn scale.

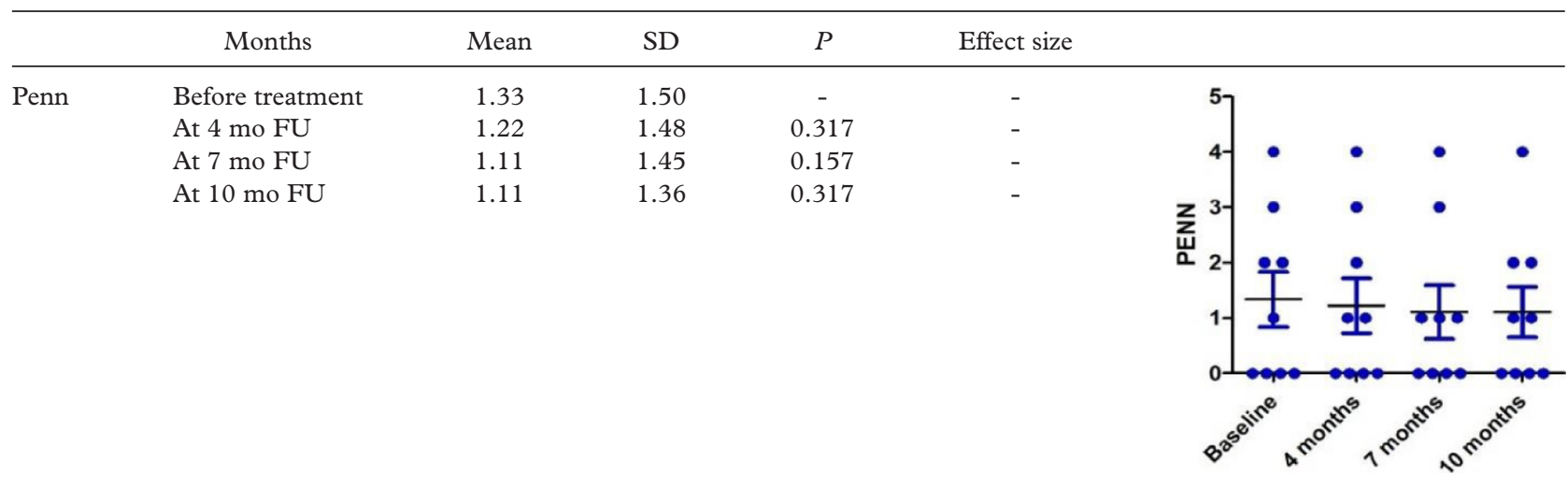

Wilcoxon test was applied comparing each month with baseline. Tendency to improvement was observed but without statistical significance.

micturition, which was not present in any patient previous to cell therapy, was recorded in six patients $(66.6 \%)$ at the end of the follow-up. At this time, five patients $(55.5 \%)$ improved in first sensation at filling, three patients $(33.3 \%)$ improved in maximum cystometric capacity, five patients $(55.5 \%)$ showed decrease in detrusor pressure at filling and six patients $(66.6 \%)$ improved in postmictional residue. Furthermore, six patients (66.6\%) improved in bladder compliance.

Table V. Scores in VAS scale.

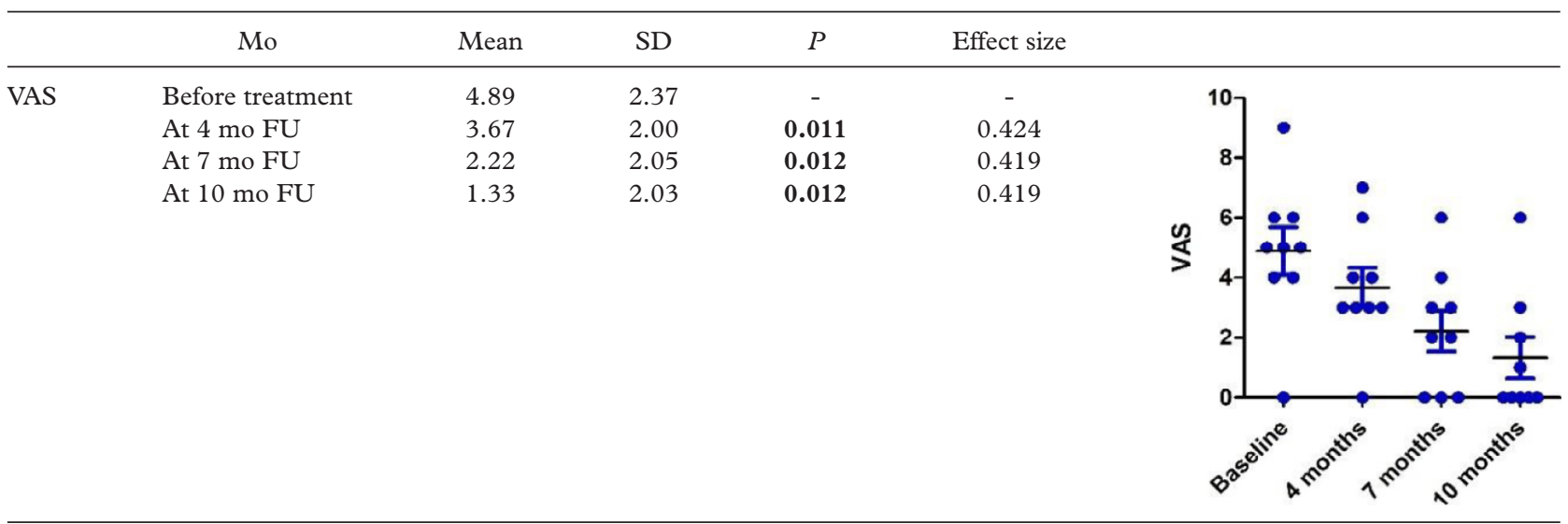

Significant $P$ values are highlighted in bold. Wilcoxon test was applied comparing each month with baseline.

Table VI. Scores in Geffner scale.

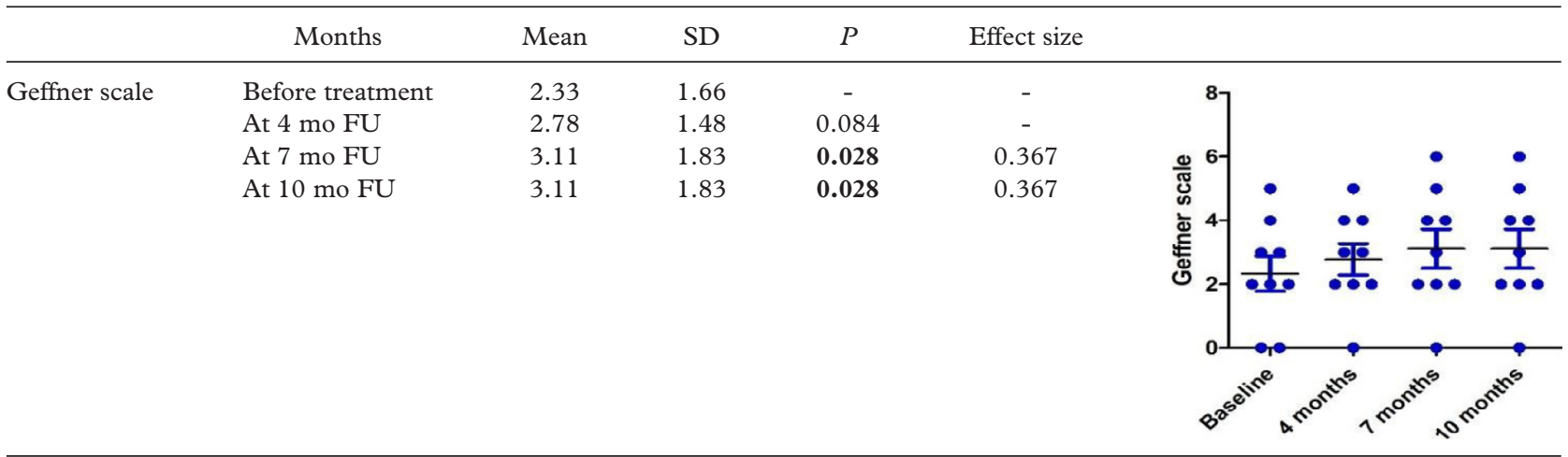

Significant $P$ values are highlighted in bold. Wilcoxon test was applied comparing each month with baseline. 
Table VII. Neurogenic bowel.

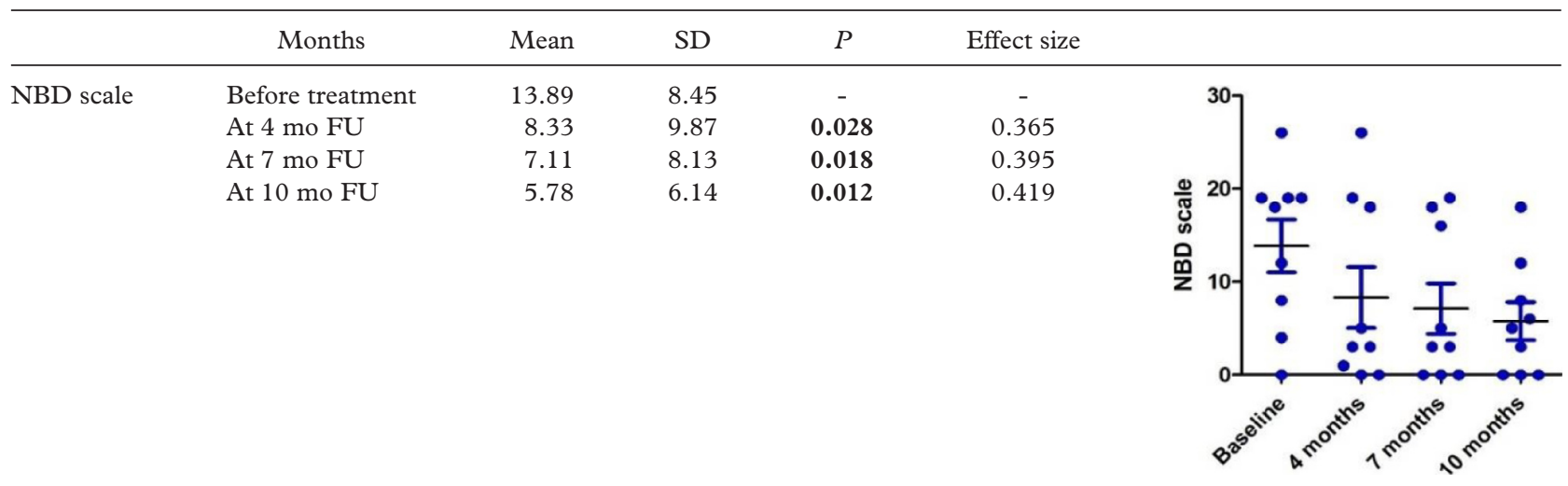

Significant $P$ values are highlighted in bold. Wilcoxon test was applied comparing each month with baseline.

\section{Neurophysiological findings}

At the end of follow-up, seven patients showed improvement in the somatosensory-evoked potentials (SSEPs) or motor-evoked potentials (MEPs) obtained prior to cell therapy. Four patients showed improvement in peripheral nerve conduction, and four patients showed improvement in voluntary muscle contraction. Moreover, infralesional polyphasic motor potentials, considered typical of active muscle reinnervation, were recorded in these four patients (Table IX).

\section{Neuroimaging studies}

Neuroimaging studies were performed before cell therapy in all of the patients in the study (Figure 6).
At the end of the follow-up, magnetic resonance studies failed to show changes in the morphology of SCI.

\section{Discussion}

Our present results confirm the benefit of intrathecal administration of autologous MSCs in patients with SCI. Only one patient in the series (patient 09) failed to obtain clinical improvement, but when a new evaluation of the patient's initial MR images was made, at the end of the study, we observed that the artifacts due to vertebral fixation device did not allow us to see clearly a zone of extreme spinal cord atrophy, which should have made us suspect a possible ineffectiveness of the treatment. This finding supports the need for a careful evaluation of the morphology of SCI

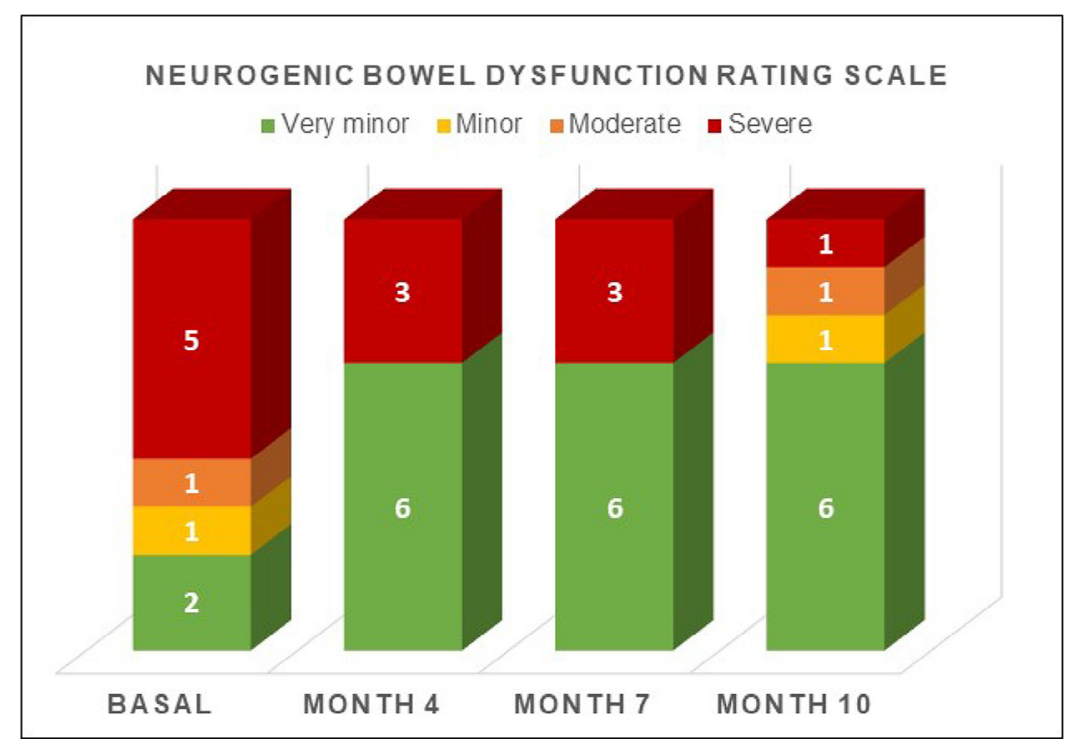

Figure 4. Improvement in NBD. Prior to cell therapy, and according to the NBD rating scale, five patients had severe NBD, one patient had minor NBD, one patient has moderate NBD and two patients had very minor dysfynction. At the end of the study, only one patient had severe NBD, one patient had moderate NBD, one patient had minor NBD and six patients had very minor NBD. 


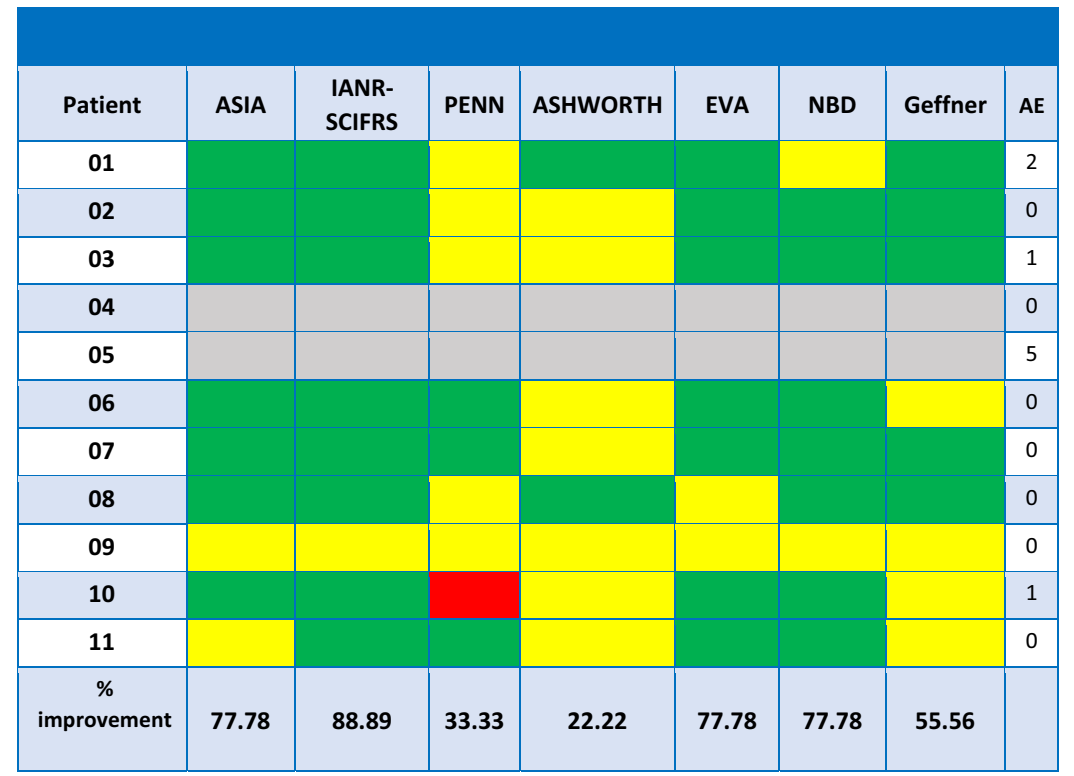

Figure 5. Improvements obtained in the patients: green, patients who improved; yellow, patients who neither improved nor worsened; red, patients who worsened; grey, patients not included in the Per Protocol set.

prior to cell therapy and, in our opinion, that efficacy should not be expected if there is no acceptable continuity of the spinal cord in the zone of SCI $[12,13]$. Despite the absence of clinical improvement, improvement in urodynamic variables and a slight improvement in sensitivity of nerve conduction were found in this patient.

In previous studies, we obtained data suggesting that, at least in some efficacy variables, the number of cells influences the results after cell therapy [11]

Table VIII. Urodynamic studies after cell therapy.

\begin{tabular}{|c|c|c|c|c|c|c|c|c|c|c|}
\hline Patient & 01 & 02 & 03 & 06 & 07 & 08 & 09 & 10 & 11 & $\%$ \\
\hline VOLUNTARY MICTURITION & YES & NO & YES & NO & YES & YES & YES & YES & NO & 66.6 \\
\hline IMPROVEMENT IN FIRS & NO & YES & YES & $\mathrm{NO}$ & NO & NO & YES & YES & YES & 55.5 \\
\hline INCREASE IN BLADDER CAP & YES & NO & NO & NO & YES & YES & NO & NO & NO & 33.3 \\
\hline DECREASE IN DETRUSOR PRESSURE AT FILLING & NO & NO & NO & NO & YES & YES & YES & YES & YES & 55.5 \\
\hline INCREASE IN BLADDER COMPLIANCE AT FILLING & YES & NO & NO & NO & YES & YES & YES & YES & YES & 66.6 \\
\hline DECREASE IN POSTMICTIONAL RESIDUE & NO & YES & YES & YES & NO & NO & YES & YES & YES & 66.6 \\
\hline
\end{tabular}

Summary of the urodynamic data after cell therapy (10 mo of follow-up). According to these studies, all patients showed improvement in some of the parameters studied, with better bladder compliance and decrease in postmictional residue in $66.6 \%$ of the cases.

Table IX. Neurophysiological studies.

\begin{tabular}{|c|c|c|c|c|c|c|c|c|c|c|}
\hline Patient & 01 & 02 & 03 & 06 & 07 & 08 & 09 & 10 & 11 & $\%$ \\
\hline IMPROVEMENT IN SSEPs & YES & YES & YES & YES & NO & NO & $\mathrm{NO}$ & $\mathrm{NO}$ & YES & 55.5 \\
\hline IMPROVEMENT IN MEPS & NO & YES & YES & YES & $\mathrm{YES}^{\star}$ & NO & NO & $\mathrm{YES}^{\star}$ & NO & 55.5 \\
\hline IMPROVEMENT IN BOTH SSEPS AND MEPS & NO & YES & YES & YES & NO & NO & NO & NO & NO & 33.3 \\
\hline IMPROVEMENT IN SENSITIVE CONDUCTION & NO & NO & YES & NO & YES & NO & YES & NO & NO & 33.3 \\
\hline IMPROVEMENT IN MOTOR CONDUCTION & NO & NO & NO & YES & YES & NO & NO & NO & NO & 22.2 \\
\hline IMPROVEMENT IN VOLUNTARY MUSCLE CONTRACTION & YES & NO & YES & YES & YES & NO & NO & NO & NO & 44.4 \\
\hline INFRALESIONAL ACTIVE MUSCLE REINNERVATION & YES & NO & YES & YES & YES & NO & NO & NO & $\mathrm{NO}$ & 44.4 \\
\hline
\end{tabular}

Summary of the neurophysiological data after cell therapy (10 mo of follow-up). In the baseline studies, all patients showed bilateral SSEPs, with variable parameters of amplitude and latency that improved at the end of the study in five patients (55.5\%). Prior to cell therapy, all patients showed MEPs that improved at the end of the study in five cases (55.5\%). In two patients ( ${ }^{\star}$ ) baseline MEPs were not obtained on the left side, but in these patients bilateral MEPs were identified at the end of follow-up. Improvement in both SSEPs and MEPs were obtained in three patients $(33.3 \%)$. 

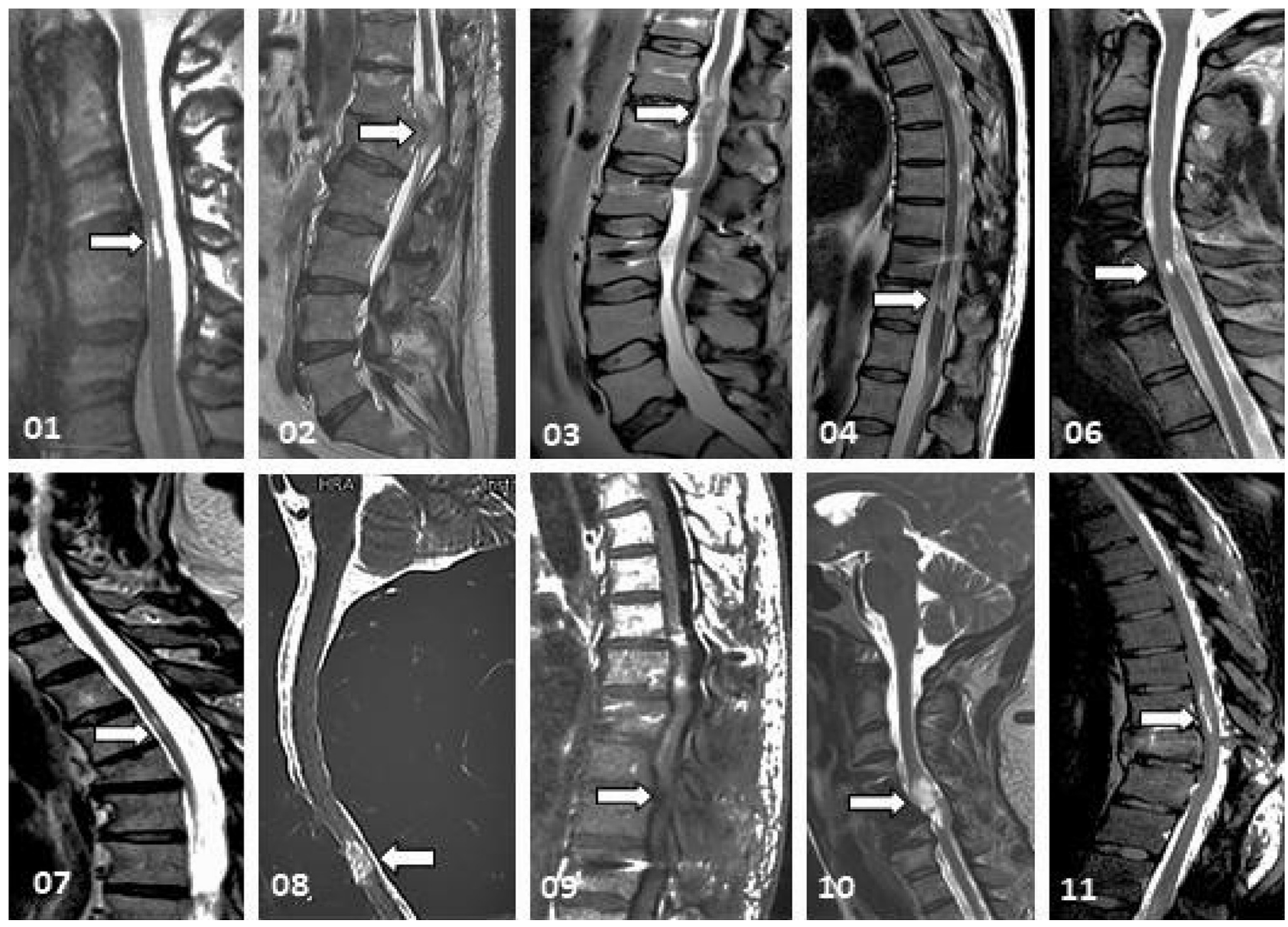

Figure 6. Aspect of the lesions (arrows) previous to cell therapy, observed in MRI, in the patients of the series.

but, at present, there is still no evidence about which are the best guidelines for MSC administration in SCI. The number of administrations has not been studied to know if a single administration can obtain the same results as repeated administrations, although the convenience of repeated administrations has been suggested $[11,12]$, and, in the statistical study of our results, when differences between doses were considered, Wilcoxon tests suggest that at least two doses of MSCs are needed to show a significant benefit in ASIA, IANRSCIFRS, VAS and NBD scores. However, the apparent absence of significant AEs after this type of cell therapy suggests that a high number of cells can be administered locally or by intrathecal route without risk, and, in terms of efficiency, a reduction in the number of administrations allows one to reduce costs, by decreasing the necessary quality controls that must be performed with each release of the cell therapy medicament.

In the present study, we explored the efficacy of three administrations of 100 million autologous MSCs every 3 months until a total of 300 million cells is reached (100/3 guideline). Despite the possible limitations of our study due to the number of patients studied and the variability of lesions, during the course of the follow-up, our patients obtained significant improvement in sensitivity, motor power, neuropathic pain and sphincter dysfunction, regardless of the level or degree of lesion, age or time elapsed from the SCI. Furthermore, our present findings showed improvements in spasticity, spasms and sexual function, although without statistical significance due to the number of patients studied. Therefore, although there are currently no data on which to base guidelines for the intrathecal administration of MSCs in patients with SCI, the present 100/3 guideline can be considered a valid option because it is safe and shows efficacy. Moreover, in the ASIA assessment, 33.3\% of our patients improved to an ASIA grade of lower disability, compared with a previous clinical trial in which we used the administration of four doses of 30 million MSCs, up to a total dose of 120 million MSCs [12]. When our present findings are compared with those described by other authors [2,3,5-10], the good results obtained, mainly in improvement of neurogenic bowel, may be due to the high number of cells administered. In any case, our present results support previous studies suggesting the safety and clinical benefit of 
intrathecal cell therapy with autologous MSCs in patients with chronic and established SCI. The results obtained in urodynamic and neurophysiological studies add an objective assessment to the clinical improvement of our patients.

\section{Conclusions}

This study supports previous evidence showing that patients with SCI can improve their neurological dysfunction after intrathecal cell therapy with autologous MSCs. In the absence of data about the best treatment guidelines, our present experience is that intrathecal administration of MSCs with a dose of 100 million cells every 3 months, up to a total of 300 million (100/3 guideline), is safe and shows benefit in patients with SCI.

\section{Acknowledgments}

The present clinical trial was mainly supported by Carlos III Institute (expedient PI14/00727) and institutions supporting the development of our cell therapy program, in particular, Mapfre and Rafael del Pino Foundations. Additional support was obtained from Atresmedia Foundation and APINME Association. We extend special thanks to Sermes Contract Research Organization for help during the development and analysis of the present study. We especially appreciate the cooperation of the Neurological CellTherapy Group from the Puerta de Hierro-Majadahonda Hospital (listed in the Supplementary Material) and the external rehabilitation team from the Lesionado Medular Foundation, and Lescer and Crene centers.

Disclosure of interests: The authors have no commercial, proprietary or financial interest in the products or companies described in this article.

\section{References}

[1] Yoon SH, Shim YS, Park YH, Chung JK, Nam JH, Kim MO, et al. Complete spinal cord injury treatment using autologous bone marrow cell transplantation and bone marrow stimulation with granulocyte macrophage-colony stimulating factor: Phase I/II clinical trial. Stem Cells 2007;25:2066-73.

[2] Parr AM, Tator CH, Keating A. Bone marrow-derived mesenchymal stromal cells for the repair of central nervous system injury. Bone Marrow Transplant 2007;40:609-19.

[3] Deda H, Inci MC, Kürekçi AE, Kayihan K, Ozgün E, Ustünsoy GE, et al. Treatment of chronic spinal cord injured patients with autologous bone marrow-derived hematopoietic stem cell transplantation: 1-year follow-up. Cytotherapy 2008;10:565-74.

[4] Vaquero J, Zurita M. Functional recovery after severe central nervous system trauma: Current perspectives for cell therapy with bone marrow stromal cells. Progr Neurobiol 2011;93: 341-9.

[5] Pal R, Venkataramana NK, Bansai A, Balaraju S, Jan M, Chandra R, et al. Ex vivo-expanded autologous bone marrow- derived mesenchymal stromal cells in human spinal cord injury/paraplegia: a pilot clinical study. Cytotherapy 2009;11:897-911.

[6] Saito F, Nakatani T, Iwase M, Maeda Y, Murao Y, Suzuki $\mathrm{Y}$, et al. Administration of cultured autologous bone marrow stromal cells into cerebrospinal fluid in spinal injury patients: a pilot study. Restor Neurol Neurosci 2012;30:127-36.

[7] Jiang PC, Xiong WP, Wang G, Ma C, Yao WQ, Kendell SF, et al. A clinical trial report of autologous bone marrow-derived mesenchymal stem cell transplantation in patients with spinal cord injury. Exp Ther Med 2013;6:140-6.

[8] Mendonça MVP, Larocca TF, Souza BS, de Freitas Souza BS, Villarreal CF, Silva LF, et al. Safety and neurological assessments after autologous transplantation of bone marrow mesenchymal stem cells in subjects with chronic spinal cord injury. Stem Cell Res Ther 2014;5:126.

[9] Karamouzian S, Nematollahi-Mahani SN, Nakhaee N, Eskandary H. Clinical safety and primary efficacy of bone marrow mesenchymal cell transplantation in subacute spinal cord injured patients. Clin Neurol Neurosurg 2012;114:935-9.

[10] Satti HS, Waheed A, Ahmed P, Ahmed K, Akram Z, Aziz T, et al. Autologous mesenchymal stromal cell transplantation for spinal cord injury: a phase I pilot study. Cytotherapy 2016;18:518-22.

[11] Vaquero J, Zurita M, Rico MA, Bonilla C, Aguayo C, Montilla $\mathrm{J}$, et al. An approach to personalized cell therapy in chronic complete paraplegia: the Puerta de Hierro phase I/II clinical trial. Cytotherapy 2016;18:1024-35.

[12] Vaquero J, Zurita M, Rico MA, Bonilla C, Aguayo C, Fernández C, Neurological Cell Therapy Group, et al. Repeated subarachnoid administrations of autologous mesenchymal stromal cells supported in autologous plasma improve quality of life in patients suffering incomplete spinal cord injury. Cytotherapy 2017;19:349-59.

[13] Vaquero J, Zurita M. Cell transplantation in paraplegic patients: The importance of properly assessing the spinal cord morphology. Clin Transplant 2013;27:968-71.

[14] World Medical Association Declaration of Helsinki. Ethical principles for medical research involving human subjects. JAMA 2000;284:3043-5.

[15] International Conference on Harmonisation Expert Working Group. ICH 22 armonized tripartite guideline: guideline for good clinical practice. June, 10, 1996. http://www.ich.org/ fileadmin/Public_Web_Site/ICH_Products/Guidelines/ Efficacy//E6/E6_R1_Guideline.pdf.

[16] Kirshblum SC, Burns SP, Biering-Sorensen F, Donovan W, Graves DE, Jha A, et al. International standards for neurological classification of spinal cord injury (revised 2011). J Spinal Cord Med 2011;34:535-46.

[17] International Association of Neurorestoratology. Spinal cord injury functional rating scale. Zhongguo Xiu Fu Chong Jian Wai Ke Za Zhi 2008;22:1021-3.

[18] Woodforde JM, Merskey H. Some relationship between subjective measures of pain. J Psychosom Res 1972;16:173-8.

[19] Penn RD, Savoy SM, Corcos D, Latash M, Gottlieb G, Parke $\mathrm{B}$, et al. Intrathecal baclofen for severe spinal spasticity. $\mathrm{N}$ Engl J Med 1989;320:1517-21.

[20] Bohannon RW, Smith MB. Interrater reliability of a modified Ashworth scale of muscle spasticity. Phys Ter 1987;67:206-7.

[21] Geffner LF, Santacruz P, Izurieta M, Flor L, Maldonado B, Auad AH, et al. Administration of autologous bone marrow stem cells into spinal cord injury patients via multiple routes is safe and improves their quality of life: comprehensive case studies. Cell Transplant 2008;17:1277-93.

[22] Krogh K, Christensen P, Sabroe S, Laurberg S. Neurogenic bowel dysfunction score. Spinal Cord 2006;44:625-31.

[23] Cohen J. A power primer. Psychol Bull 1992;112:155-9. 
[24] R Core Team. R: A language and environment for statistical computing. Vienna, Austria.: R Foundation for Statistical Computing; 2017. AVAILABLE FROM: https://www.Rproject.org/.

[25] Wickham H. ggplot2: Elegant Graphics for Data Analysis. Springer-Verlag New York; 2009.

[26] International Conference on Harmonisation of Technical Requirements for Registration of Pharmaceuticals for Human Use. Statistical principles for clinical trials E9. 1998. AVAILABLE FROM: http://www.ich.org/ products/guidelines/efficacy/efficacy-single/article/statistical -principlesfor-clinical-trials.html.

\section{Appendix: Supplementary material}

Supplementary data to this article can be found online at doi:10.1016/j.jcyt.2018.03.032. 